\title{
El papel mediador de la regulación emocional entre el juego patológico, uso abusivo de Internet y videojuegos y la sintomatología disfuncional en jóvenes y adolescentes
}

\author{
Mediating role of emotional regulation between impulsive \\ behavior in gambling, Internet and videogame abuse, and \\ dysfunctional symptomatology in young adults and adolescents.
}

\author{
Ana Estévez Gutiérrez*, David Herrero Fernández*, Izaskun Sarabia Gonzalvo*, Paula \\ Jáuregui Bilbao*
}

*Universidad de Deusto

\section{Resumen}

La manera de regular las emociones podría estar relacionada con la realización de conductas impulsivas de riesgo en jóvenes y adolescentes, por lo que su estudio sería de gran relevancia. Algunas de las conductas de riesgo menos estudiadas son el juego patológico, el abuso de Internet y videojuegos. Los objetivos de esta investigación han sido conocer la relación existente entre estas conductas, la regulación de las emociones, y la sintomatología psicológica disfuncional (depresión, ansiedad, ansiedad fóbica, somatización, obsesión-compulsión, sensibilidad interpersonal, ira-hostilidad, ideación paranoide, y psicoticismo). Asimismo, se ha estudiado si la regulación emocional media entre el juego patológico, el abuso de Internet y videojuegos y la aparición de síntomas psicológicos. La muestra ha estado compuesta por 1312 jóvenes y adolescentes de entre 12 y 30 años, procedentes de centros escolares, universitarios y grupos de tiempo libre, y de asociaciones y centros asociados a la FEJAR (Federación Española de Jugadores de Azar Rehabilitados). Los participantes han completado medidas de conducta impulsiva, regulación emocional, y sintomatología disfuncional. Los resultados han mostrado que existe una relación positiva y significativa entre estas variables de manera general. Asimismo, han señalado que la regulación emocional media de manera total y parcial en la aparición de sintomatología disfuncional en jóvenes y adolescentes que tienen problemáticas en estas conductas impulsivas, a excepción de la relación entre abuso de videojuegos y sintomatología depresiva. El entrenamiento en habilidades de regulación emocional podría ser útil para abordar estas conductas en jóvenes y adolescentes. Palabras Clave:juego patológico, abuso, Internet, videojuegos, regulación emocional, jóvenes, adolescencia, sintomatología disfuncional.

\begin{abstract}
The way emotions are regulated might affect the engagement on risk behaviors in adolescents and young adults. Therefore, studying the relationship between these variables could be of great importance. Some of the less studied risky behaviors are pathological gambling, and Internet and videogame abuse. This research aims to analyze the existing relationship between such risky behaviors, emotion regulation, and dysfunctional psychological symptomatology (depression, anxiety, phobic anxiety, somatization, obsessive-compulsive behavior, interpersonal sensitivity, hostility, paranoid ideation, and psychoticism). In addition, it also looks to assess whether emotional regulation plays a mediating role between pathological gambling, and Internet and videogame abuse, and psychological symptomatology. The sample was composed of 1312 young adults and adolescents, aged between 12 and 30, recruited from scholar centers, universities and free time groups, and from associations and centers associated with FEJAR (Spanish Federation of Rehabilitated Gamblers). Participants completed measurements of impulsive behavior, emotion regulation, and dysfunctional symptomatology. Results showed that there is generally a positive and significant relation between these variables. Moreover, it has been pointed out that emotion regulation mediates the association between impulsive behavior and dysfunctional symptomatology among those young adults and adolescents who engage in these impulsive behaviors, except for the relation between videogame abuse and depressive symptomatology. Training in emotional regulation skills could be useful in dealing with and treating this type of behaviors in adolescents and young adults. Key Words: pathological gambling, abuse, Internet, videogames, emotion regulation, young adults, adolescence.
\end{abstract}

Recibido: Octubre 2013; Aceptado: Enero 2014

Enviar correspondencia a:

Ana Estévez. Departamento de Psicología. Universidad de Deusto. Apartado 1, 48080, Bilbao, España; E-mail: aestevez@deusto.es 


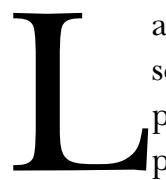

a adolescencia constituye un período donde se aprenden, construyen y/o desarrollan comportamientos diferentes a los de la infancia que pueden suponer algún tipo de peligro o perjuicio (García del Castillo y Días, 2007; Gibbons, Kingsbury, y Gerrard, 2012; Maguire, Wells, y Katz, 2011). Numerosas investigaciones han estudiado las conductas de riesgo en jóvenes y adolescentes, entre las que destacan las relacionadas con el control de los impulsos tales como el juego patológico (Donati, Chiesi, y Primi, 2013), uso problemático de videojuegos (Carbonell, 2014; Walther, Morgenstern, y Hanewinkel, 2012) e Internet (Kaess et al., 2014; Tsitsika, Critselis, Janikian, Kormas, y Kafetzis, 2011) y que se han asociado con problemas a nivel relacional, económico y de rendimiento escolar (Hastings et al., 2009; Raisamo, Halme, Murto, y Lintonen, 2013; Ruiz-Olivares, Lucena, Pino, y Herruzo, 2010). A pesar de que su prevalencia es alta en esta etapa vital, de entre el 3,7\% y el 9,9\% en el caso de Internet (Carbonell, Fúster, Chamarro, y Oberst, 2012; Durkee et al., 2012; Puerta-Cortés y Carbonell, 2014); del 3,9\% en juego patológico (Petry, 2006) y del 3\% en videojuegos (Van Rooij, Schoenmakers, Vermulst, Van Den Eijnden, y Van De Mheen, 2011), son conductas pocos estudiadas (Karim y Chaudhri, 2012).

Las dificultades para controlar los impulsos se han relacionado con factores como la dificultad para la regulación emocional (Schreiber, Grant y Oudlaug, 2012; Tice, Bratslavsky y Baumeister, 2001). Por ejemplo, en el caso de las adicciones con sustancias, se ha encontrado una mayor probabilidad de abuso de las mismas en jóvenes con mala regulación emocional (Hessler y Katz, 2010). En el juego patológico, algunos estudios han relacionado el juego con la expectativa de conseguir estados anímicos positivos o aliviar estados anímicos negativos (Shead, Callan, y Hodgins, 2008), y se ha encontrado que existirían dificultades en regulación emocional en personas adultas que están en tratamiento por problemas de juego patológico (Williams, Geisham, Eerskine y Cassedy, 2012). En jóvenes y adolescentes, apenas existen estudios que analicen la regulación emocional y el juego patológico. En otras conductas impulsivas, los estudios al respecto también son muy escasos y apenas han sido estudiadas en población joven y adolescente. Por ejemplo, se han encontrado evidencias de que la regulación emocional podría estar interviniendo en la aparición de un uso compulsivo de los videojuegos (Haagsma, Caplan, Peters, y Pieterse, 2013), así como en el caso de Internet, en el que se ha encontrado que la motivación de aliviar sentimientos de distrés estaría ligada con su uso compulsivo (Caplan, 2010). Aunque estos estudios apuntan a una relación existente entre la regulación emocional y las conductas impulsivas en la juventud y adolescencia, sin embargo, otros como el de Miller, Vachon, y Aalsma (2012), en el que participaron adolescentes con problemas con la justicia, sugirieron que la falta de regulación emocional no estaba asociada con comportamientos impulsivos en el caso de la conducta sexual. Por tanto, es necesario seguir estudiando la relación entre conducta impulsiva y regulación emocional en jóvenes y adolescentes.

Unido a esto, la falta de regulación emocional es un proceso esencial para el funcionamiento adaptativo, de tal modo que la presencia de dificultades para regular las emociones se relacionaría con la etiología y mantenimiento de la mayoría de las formas de psicopatología (Bradley, 2000; Silk, Steinberg, y Morris, 2003). En el caso de los jóvenes y adolescentes, la mala regulación emocional ha sido considerada un factor que aumenta el riesgo de un amplio rango de respuestas psicopatológicas (McLaughlin, Hatzenbuehler, Mennin, y Nolen-Hoeksema, 2011), tales como problemas de conducta (Mullin y Hinshaw, 2007; Southam-Gerow y Kendall, 2002), conductas externalizantes y de riesgo (Cooper, Agocha, y Sheldon, 2000; Cooper, Wood, Orcutt, y Albino, 2003; Curry y Youngblade, 2006; Silk et al., 2003), sintomatología depresiva (Betts, Gullone, y Allen, 2009) o intentos de suicidio (Pisani et al., 2013), entre otras.

Como puede verse, sería necesario conocer el papel que juega la regulación emocional en la relación entre las conductas impulsivas de juego patológico, Internet y videojuegos en la juventud y adolescencia y la aparición de sintomatología disfuncional. En este sentido, se ha encontrado que la regulación emocional tendría un papel mediador entre la impulsividad y la depresión en adolescentes (d'Acremont y Van der Linden, 2007), pero se desconoce si la regulación emocional podría mediar entre el juego patológico, el abuso de Internet, y videojuegos, y la aparición posterior de sintomatología disfuncional.

Por ello, los objetivos de este estudio han sido los siguientes. En primer lugar, analizar la relación existente entre la regulación emocional y las conductas impulsivas de juego patológico, uso abusivo de Internet y videojuegos en jóvenes y adolescentes. En segundo lugar, analizar la relación existente entre regulación emocional y sintomatología disfuncional en ese periodo vital. Y, por último, valorar si la regulación emocional interviene como mediadora entre estas conductas y la aparición de sintomatología disfuncional.

\section{Método}

\section{Muestra}

La muestra total de este estudio se compone de 1316 jóvenes y adolescentes de entre 12 y 30 años $(\mathrm{M}=17.28, D T$ $=2.70$ ), siendo el $57.4 \%$ hombres y el $42.6 \%$ mujeres. Más concretamente, 321 participantes $(24.3 \%)$ tenían entre 12 y 15 años; 837 (63.4\%) tenían entre 16 y 20 años; 141 (10.7\%) tenían entre 21 y 25 años, y $17(1.3 \%)$ tenían entre 26 y 30 años. Finalmente, cuatro participantes $(0.3 \%)$ no informaron de la edad. Por otro lado, los participantes procedían de centros escolares, universitarios y grupos de tiempo libre, y de asociaciones y centros asociados a la FEJAR (Federación Es- 
pañola de Jugadores de Azar Rehabilitados). Según el MULTICAGE, 25 de los participantes $(1.9 \%)$ tenían problemas de juego; $301(22.8 \%)$ tenían problemas relacionados con el uso de Internet, y 129 (9.7\%) tenían problemas con el uso de videojuegos. En cuanto a la situación laboral, mayoritariamente eran estudiantes $(96.2 \%)$, seguido de personas que estudian y trabajan $(2.1 \%)$, personas en activo $(1 \%)$ y personas en situación de desempleo $(0.7 \%)$. El nivel de estudios de este grupo es la siguiente: estudios secundarios (58.5\%), Formación Profesional (18.7\%), estudios primarios (15.6\%), estudios universitarios $(6,8 \%)$ y sin estudios $(0.5 \%)$.

\section{Instrumentos}

Conducta impulsiva. MULTICAGE CAD-4 (Pedrero Pérez et al., 2007). Es un instrumento para valorar conductas adictivas, con o sin sustancia. Está destinado a hombres y mujeres de entre 14 y 90 años. Consta de 32 ítems, que se agrupan en ocho factores: abuso o dependencia del alcohol, juego patológico, adicción a sustancias, trastornos de la alimentación, abuso a Internet, abuso a videojuegos, gasto compulsivo y conducta sexual impulsiva. Los resultados se clasifican en las siguientes categorías: Inexistencia del problema (de 0 a 1 punto), Posible existencia del problema (2 puntos), Muy probable existencia del problema (3 puntos), y Segura existencia del problema (4 puntos). Los valores de consistencia interna son satisfactorios (alfa de Cronbach de la escala total $=.86$; las subescalas muestran valores superiores a .70). La fiabilidad test-retest a los 20 días fue de $r=.89$. La validez de criterio también es adecuada (permite detectar entre el 90 y el $100 \%$ de los casos ya diagnosticados), así como la validez de constructo (el análisis factorial exploratorio identifica como independientes las 8 escalas propuestas, que explican en conjunto el $63.8 \%$ de la varianza total). En nuestra muestra, el alfa de Cronbach de la escala total fue de .69. En este estudio, se han utilizado las subescalas de juego patológico (alfa de Cronbach $=.71$ ), adicción a Internet (alfa de Cronbach $=.71$ ), y adicción a videojuegos (alfa de Cronbach = .76).

Regulación Emocional. Escala de Dificultades de Regulación Emocional (DERS; Gratz y Roemer, 2004) Es un cuestionario que mide dificultades de regulación emocional, a través de cinco factores: Descontrol Emocional, Rechazo Emocional, Interferencia Cotidiana, Desatención Emocional, y Confusión Emocional. Más concretamente, la escala del Descontrol Emocional consiste en una sensación de desbordamiento debido a la intensidad emocional y la sensación de persistencia de los estados emocionales negativos. Su interferencia con la vida se muestra en la escala Interferencia cotidiana. La escala de Rechazo Emocional evalúa la tendencia de la persona a juzgar de forma negativa su propia experiencia emocional y, como consecuencia, a reaccionar con vergüenza o con malestar ante sus propias emociones. La Confusión Emocional está referida a la incapacidad del individuo para nombrar con claridad sus emociones y, por último, la Desatención Emocional, se define como la incapacidad para dedicar recursos atencionales a la información atencional. Se responde a través de una escala Likert de cinco grados, y consta de 28 ítems que miden la frecuencia con la que ocurren las afirmaciones en la vida del sujeto. Los valores de consistencia interna en la adaptación española (Hervás y Jódar, 2008) son satisfactorios (Alfa de la escala total $=.93$; el valor de Alfa en las distintas subescalas oscila entre .73 y .91). En nuestro estudio, el valor del alfa de Cronbach fue de .91.

Sintomatología Disfuncional. Symptom Checklist-90-Revised (SCL-90-R; Derogatis, 2002). Es un instrumento multidimensional que cuestiona al individuo sobre la existencia e intensidad de 90 síntomas psiquiátricos y psicosomáticos. Se responde a través de una escala Likert de 0 (el síntoma no me molesta en absoluto) a 4 (me molesta de manera extrema), en función de la molestia que le hayan ocasionado en los últimos siete días. Explora nueve factores o dimensiones sintomáticas: somatización, obsesión-compulsión, sensibilidad interpersonal, depresión, ansiedad, ira-hostilidad, ansiedad fóbica, ideación paranoide, y psicoticismo. Está destinado a personas de entre 13 y 65 años de edad. En cuanto a su fiabilidad, los coeficientes del Alfa de Cronbach de este instrumento oscilan entre .77 y .90 . En nuestro estudio, el alfa de Cronbach fue de .97 .

\section{Procedimiento}

La realización del estudio ha sido precedida por una carta explicativa que contenía los aspectos que a continuación se detallan: entidad que solicita y que realiza el estudio; contenido y objetivos del estudio; duración y aspectos que se van a medir; posibilidad de realizar el estudio tanto en papel como a través de Internet; metodología de recogida de cuestionarios; consentimiento informado; voluntariedad de la realización del estudio; confidencialidad y anonimato de los datos obtenidos y teléfono y mails de los investigadores de referencia para poder contactar.

Los cuestionarios se han recogido tanto en formato de papel como a través de un cuestionario on line. En el caso de los cuestionarios de papel, en la mayoría de los casos, los investigadores del estudio se han desplazado a los centros para recoger los datos. En relación al cuestionario on line, el contenido de las preguntas ha sido el mismo que el utilizado en papel. Este estudio ha recibido el certificado de idoneidad ética por parte de la Universidad.

\section{Diseño y Estrategia de Análisis}

Se llevó a cabo un diseño de tipo transversal correlacional. En cuanto a los análisis, se llevaron a cabo con el paquete estadístico SPSS 17.0. Primeramente se calcularon los coeficientes de correlación $r$ de Pearson entre todas las variables, a fin de identificar las que podían ser incluidas, en la segunda parte, en los análisis de mediación. El criterio de 
selección fue la significación estadística en las correlaciones bivariadas. En cuanto a los análisis de mediación, se utilizó la macro Process para SPSS (Hayes, 2013). En cada uno de los modelos de mediación analizados, se informa del efecto total y directo, incluyendo el valor del coeficiente beta y de su significación. En los casos en que el coeficiente beta mantiene su significación en el efecto directo, se añade el test $Z$ de Sobel, a fin de analizar la existencia de un posible efecto parcial de mediación.

De esta forma, los análisis se dividen en dos partes. En la primera parte se analizaron las correlaciones entre los tres bloques de variables (conductas impulsivas, regulación emocional, y sintomatología), a fin de identificar las que podrían ser incluidas en los posteriores modelos de mediación. En la segunda parte, tras verificar la asociación bivariada entre las variables, se procedió a analizar el papel mediador de la regulación emocional entre el juego patológico, el abuso de Internet y de videojuegos, que fueron tomadas como variables independientes, y la sintomatología valorada (ansiedad, ansiedad fóbica somatización, comportamiento obsesivo-compulsivo, sensibilidad interpersonal, depresión, ira-hostilidad, ideación paranoide, y psicoticismo), que fueron analizadas como variables dependientes. De esta forma, en cada modelo de mediación se introdujeron simultáneamente todos los posibles mediadores. En todos los casos se calculó el test $Z$ de Sobel, a fin de determinar la influencia individual de cada mediador en el efecto hallado. En cada uno de los modelos de mediación ejecutados se suprimieron las variables que no habían mostrado correlación significativa con la variable independiente en cuestión, al ser condición necesaria para poder llevar a cabo este análisis.

\section{Resultados}

\section{Análisis correlacional bivariado}

En primer lugar, los resultados relativos a la asociación entre los comportamientos impulsivos y la regulación emocional se exponen en la Tabla 1. Tal como se puede observar, en general las variables relativas a conductas impulsivas estuvieron positiva y significativamente relacionadas con la regulación emocional. De las tres conductas impulsivas valoradas, la relativa al uso de los videojuegos fue la que mostró correlaciones más débiles.

Por otro lado la asociación entre los comportamientos impulsivos y la sintomatología se detalla en la Tabla 2. Los resultados muestran que ambos bloques de variables están positiva y significativamente asociados, de manera que el único coeficiente que no alcanzó la significación fue el que relacionó el uso de videojuegos con la depresión.

Finalmente, en la Tabla 3 se muestra la asociación entre la regulación emocional y la sintomatología. Al igual que en el caso anterior, la mayoría de los coeficientes fueron positivos y significativos, siendo la desatención la variable que mostró coeficientes más bajos con la sintomatología valorada.

\section{Análisis de mediación}

En base a los resultados obtenidos en el estudio correlacional bivariado se llevaron a cabo los análisis de mediación. De este modo, en la Tabla 4 se detallan los resultados de las mediaciones relativas a las variables independientes consideradas: conducta impulsiva de juego patológico, conducta impulsiva de uso de Internet, y conducta impulsiva de uso de videojuegos.

Tabla 1

Correlaciones entre las formas de conductas impulsivas y la regulación emocional

\begin{tabular}{lcccccc}
\hline & $\begin{array}{c}\text { Descontrol } \\
\text { Emocional }\end{array}$ & Rechazo & Interferencia & Desatención & Confusión & $\begin{array}{c}\text { Total Regulación } \\
\text { Emocional }\end{array}$ \\
\hline Juego patológico & $.11^{* * *}$ & $.14^{* * *}$ & -.01 & $.09^{* * *}$ & $.07^{* *}$ & $.13^{* * *}$ \\
Internet & $.18^{* * *}$ & $.13^{* * *}$ & $.17^{* * *}$ & .00 & $.16^{* * *}$ & $.19^{* * *}$ \\
Videojuegos & $.06^{*}$ & .03 & .03 & .05 & $.06^{*}$ & $.07^{*}$ \\
\hline
\end{tabular}

${ }^{*} p<.05,{ }^{* *} p<.01,{ }^{* * *} p<.001$.

Tabla 2

Correlaciones entre las formas de conductas impulsivas y la sintomatología

\begin{tabular}{|c|c|c|c|c|c|c|c|c|c|}
\hline & Ansiedad & $\begin{array}{c}\text { Ansiedad } \\
\text { Fóbica }\end{array}$ & Somatiz. & Obs.-Comp. & $\begin{array}{l}\text { Sens. } \\
\text { Interp. }\end{array}$ & Depres. & Hostil. & Pens. Paran. & Psicotic. \\
\hline Juego patológico & $.15^{* * *}$ & $.16^{* * *}$ & $.10 * * *$ & $.09 * * *$ & $.09 * * *$ & $.15^{* * *}$ & $.10 * * *$ & $.12^{* * *}$ & $.15^{* * *}$ \\
\hline Internet & $.16^{* * *}$ & $.10 * * *$ & $.12^{* * *}$ & $.19 * * *$ & $.20 * * *$ & $.19 * * *$ & $.23^{* * *}$ & $.23^{* * *}$ & $.20 * * *$ \\
\hline Videojuegos & $.08^{* *}$ & $.12^{* * *}$ & $.06 *$ & $.11^{* * *}$ & $.07 * *$ & .05 & $.12^{* * *}$ & $.11^{* * *}$ & $.11^{* * *}$ \\
\hline
\end{tabular}

Nota. "Somatiz": Somatización, “Obs.-Comp.": Obsesión-Compulsión, Sens.Interp.": Sensibilidad Interpersonal, “Depres.": Depresión,

"Hostil.": Hostilidad, "Pens. Paran.": Pensamiento Paranoide, "Psicotic.": Psicoticismo.

${ }^{*} p<.05,{ }^{* *} p<.01,{ }^{* *} p<.001$ 
Tabla 3

Correlaciones entre la regulación emocional y la sintomatología

\begin{tabular}{|c|c|c|c|c|c|c|c|c|c|}
\hline & Ansiedad & $\begin{array}{l}\text { Ansiedad } \\
\text { Fóbica }\end{array}$ & Somatiz. & $\begin{array}{l}\text { Obs.- } \\
\text { Comp. }\end{array}$ & $\begin{array}{l}\text { Sens. } \\
\text { Interp. }\end{array}$ & Depres. & Hostil. & $\begin{array}{l}\text { Pens. } \\
\text { Paran. }\end{array}$ & Psicotic. \\
\hline Desc. Emocional & $.50 * * *$ & $.34^{* * *}$ & $.41 * * *$ & $.50 * * *$ & $.41 * * *$ & $.53^{* * *}$ & $.54^{* * *}$ & $.42 * * *$ & $.49 * * *$ \\
\hline Rechazo & $.41 * * *$ & $.30 * * *$ & $.35^{* * *}$ & $.43^{* * *}$ & $.43 * * *$ & $.51 * * *$ & $.32 * * *$ & $.39 * * *$ & $.45^{* * *}$ \\
\hline Inteferencia & $.40^{* * *}$ & $.24^{* * *}$ & $.36^{* * *}$ & $.50^{* * *}$ & $.40 * * *$ & $.50^{* * *}$ & $.38 * * *$ & $.37^{* * *}$ & $.38 * * *$ \\
\hline Desatención & .04 & $.11^{* * *}$ & $.06^{*}$ & .04 & $.07^{*}$ & .05 & $.08^{* *}$ & .03 & $.08 * *$ \\
\hline Confusión & $.33^{* * *}$ & $.28^{* * *}$ & $.32 * * *$ & $.36^{* * *}$ & $.36^{* * *}$ & $.40 * * *$ & $.27 * * *$ & $.27 * * *$ & $.38 * * *$ \\
\hline $\begin{array}{l}\text { Total Regulación } \\
\text { Emocional }\end{array}$ & $.52^{* * *}$ & $.38 * * *$ & $.45^{* * *}$ & $.56^{* * *}$ & $.50^{* * *}$ & $.61^{* * *}$ & $.50 * * *$ & $.46^{* * *}$ & $.54^{* * *}$ \\
\hline
\end{tabular}

Nota. “Somatiz”: Somatización, “Obs.-Comp.": Obsesión-Compulsión, Sens. Interp.”: Sensibilidad Interpersonal, “Depres.”: Depresión,

"Hostil.": Hostilidad, "Pens. Paran.": Pensamiento Paranoide, "Psicotic.": Psicoticismo.

${ }^{*} p<.05,{ }^{* *} p<.01,{ }^{* * *} p<.001$.

En el caso de la conducta impulsiva de juego patológico, tal como se puede observar, en cinco de los casos hubo mediación parcial de la regulación emocional entre el juego patológico y las variables ansiedad, ansiedad fóbica, depresión, pensamiento paranoide, y psicoticismo, mientras que se observaron mediaciones perfectas en los casos de los síntomas relativos a la somatización, conducta obsesivo-compulsiva, sensibilidad interpersonal, y hostilidad. En general, la variable que menor efecto mediador tuvo fue la desatención, mientras que las variables descontrol emocional, rechazo, y confusión evidenciaron un efecto significativo en las mediaciones, de acuerdo con los resultados del test de Sobel.

En el caso de la conducta impulsiva de uso de Internet, tal como se puede observar en los nueve contrastes realizados se obtuvieron efectos mediacionales significativos. En el caso de la somatización se halló una mediación perfecta, mientras que en los restantes casos fue parcial. En general, los cuatro factores analizados como mediadores tuvieron efectos significativos en las mediaciones realizadas.

Finalmente, en el caso de la conducta impulsiva de uso de videojuegos, tal como se puede apreciar, en este caso sólo se tomaron como mediadores potenciales el descontrol emocional y la confusión, en base a los resultados de las correlaciones bivariadas anteriormente expuestas. $\mathrm{Al}$ igual que en los dos anteriores casos, se evidenció efecto mediacional significativo en todos los casos. En cuatro de los contrastes se halló mediación perfecta (ansiedad, somatización, sensibilidad interpersonal, y psicoticismo), mientras que en los cuatro restantes se encontró efecto de mediación parcial. En todos los casos la variable descontrol emocional tuvo un efecto más elevado en la explicación de la mediación.

\section{Discusión}

Los resultados de este estudio suponen un paso más en la profundización del papel que tiene la regulación emocional junto con las conductas impulsivas en la aparición de síntomas disfuncionales en jóvenes y adolescentes. En primer lugar, se ha estudiado la relación entre el juego patológico, el uso abusivo de Internet y videojuegos, y la regulación emocional en jóvenes y adolescentes. Como ha podido observarse, se ha encontrado relación entre ellas. Aunque son escasos los estudios realizados hasta el momento, los estudios previos van en la misma dirección y han resaltado el vínculo existente entre la regulación emocional, la impulsividad, y las conductas de abuso de sustancias y el juego patológico en este tramo de edad (Schreiber el al., 2012). Asimismo, autores como Hessler y Katz (2010) han encontrado que las personas con menor habilidad en regulación emocional en la infancia presentan en la adolescencia un nivel mayor de conductas de riesgo.

En segundo lugar, se ha analizado la relación entre la regulación emocional y la sintomatología disfuncional en jóvenes y adolescentes y se ha encontrado asociación entre ambas. Estudios recientes como el de Bender, Reinholdt-Dunne, Esbjørn, y Pons (2012); Hsieh y Stright (2012) o el de Lougheed y Hollenstein (2012) encontraron también en adolescentes que la regulación emocional se asociaba con problemas internalizantes. En el caso de niños, autores como Keenan (2000) han encontrado que la falta de regulación emocional podría ser un factor de riesgo en la psicopatología infantil. Algunos estudios longitudinales apuntan también a que los problemas en la regulación emocional precederían a la aparición de síntomas como la ansiedad o la depresión (Szwedo, 2013), lo cual pone de relieve la importancia de este proceso para el análisis de la sintomatología desadaptativa.

Por último, se ha estudiado el papel mediador de la regulación emocional entre el juego patológico, el abuso de Internet, y videojuegos, y la aparición de sintomatología disfuncional en jóvenes y adolescentes. Los resultados han mostrado la existencia de mediaciones parciales y totales 


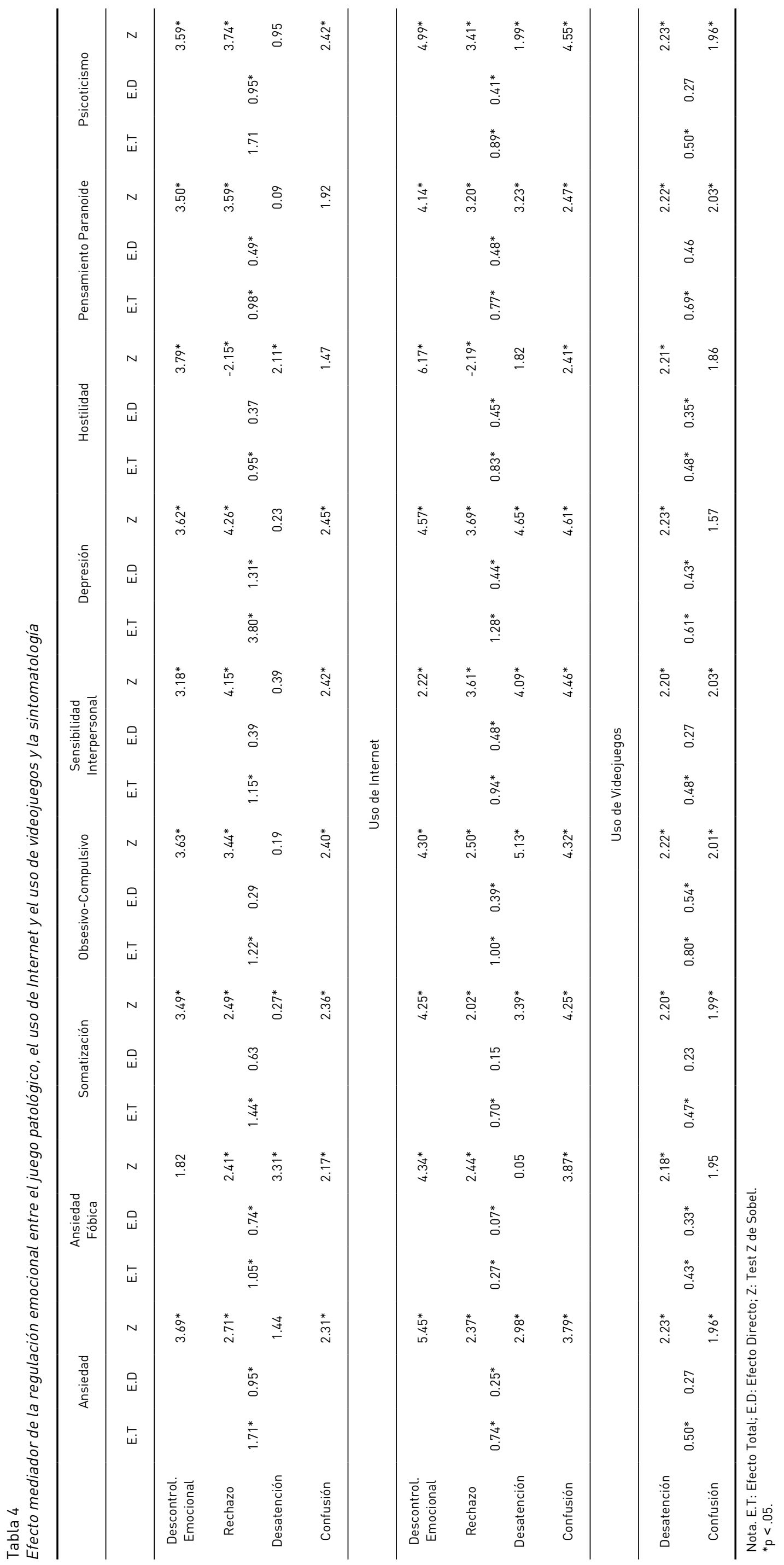


entre ambas partes, a excepción de la relación entre abuso de videojuegos y sintomatología depresiva. Como ha podido verse en los resultados obtenidos, las emociones y su regulación son aspectos muy importantes en la adolescencia y juventud, y en las conductas impulsivas previamente mencionadas. Estos resultados están en la línea de los hallazgos encontrados por Caplan (2010), Haagsma et al. (2013), y Williams et al. (2012). Unido a esto, es importante señalar que uno de los factores emocionales que más asociación ha obtenido ha sido el descontrol emocional, que como se ha detallado previamente, consiste en la sensación de desbordamiento debido a la intensidad emocional con ítems que provenían de un factor original denominado Dificultades en el control de impulsos como, por ejemplo, "cuando me siento mal, pierdo el control” (Hervás y Jódar, 2008). En este sentido aunque en el DSM-V (APA, 2013) el juego patológico se encuentra en el capítulo de adicciones comportamentales, previamente estaba catalogado dentro del apartado de control de los impulsos en el DSM-IV-TR (APA, 2001).

Este estudio, a pesar de su utilidad, no está exento de limitaciones. En primer lugar, se trata de un estudio transversal, lo que supone que no podamos concluir causalidad y no tengamos información previa de los participantes del estudio. Es necesario seguir haciendo investigaciones longitudinales para contrastar los resultados y comprender mejor los aspectos implicados en los mismos. Por otro lado, se trata de cuestionarios autoaplicados y, en algunos casos, la recogida de datos ha sido on line. Mientras que la recogida de datos on line tiene muchas ventajas y que estudios previos señalan que no existen diferencias significativas entre los cuestionarios de lápiz y papel y los cuestionarios online (Buchan, DeAngelis, y Levinson, 2005; Buchanan y Smith, 1999), los cuestionarios autoaplicados no están exentos de limitaciones. Autores como Vasiley, Crowell, Beauchaine, Mead, y Gatzke-Kopp (2009) indican que las puntuaciones obtenidas en el DERS se corresponderían con medidas fisiológicas de dificultades de regulación emocional. Por lo que la realización de estudios que incorporaran otro tipo de instrumentos, así como el empleo de medidas de diagnóstico más específicas que las herramientas de screening aquí utilizadas, contribuiría a la contrastación de los resultados obtenidos.

A modo de conclusión, se podría señalar que el entrenamiento en habilidades de regulación emocional podría ayudar en el tratamiento de la sintomatología disfuncional en jóvenes y adolescentes (Berking, Margraf, Ebert, Wupperman, Hofmann, y Junghanns, 2011). Por otro lado, también se observa que la regulación emocional tiene relevancia en conductas menos estudiadas pero de importancia cada vez mayor en jóvenes y adolescentes como son el juego patológico, previsiblemente incrementado por la aparición del juego on line (Griffiths y Parke, 2010), la conducta abusiva de videojuegos y de Internet. Como se ha señalado, la regulación emocional media la relación entre este tipo de conductas y la aparición de síntomas disfuncionales en jó- venes y adolescentes con problemática de juego patológico, el abuso de Internet y de videojuegos. Por tanto, la regulación de emociones podría ser un elemento importante de prevención y tratamiento de estas problemáticas a edades tempranas. En ese sentido, la inclusión de programas de habilidades emocionales en la intervención con esas problemáticas podría ser un elemento a evaluar para valorar la eficacia de los mismos en la intervención.

\section{Agradecimientos}

Los resultados que se presentan en el siguiente artículo han sido obtenidos gracias a la financiación de la ONCE a través de la I edición del Certamen Internacional ONCE de Investigación sobre Juego Responsable.

La muestra ha sido obtenida gracias a la ayuda de la Federación Española de Jugadores de Azar Rehabilitados.

\section{Conflicto de intereses}

Los autores declaran que no existe ningún conflicto de interés en este trabajo.

\section{Referencias}

American Psychiatric Association (2001). Manual diagnóstico y estadístico de los trastornos mentales D.S.M-IV-TR. Barcelona: Masson.

American Psychiatric Association (2013). Diagnostic and statistical manual of mental disorders (5th ed.). Arlington, VA: American Psychiatric Publishing.

Bender, P. K., Reinholdt-Dunne, M. L., Esbjørn, B. H., y Pons, F. F. (2012). Emotion dysregulation and anxiety in children and adolescents: Gender differences. Personality And Individual Differences, 53, 284-288. doi:10.1016/j. paid.2012.03.027

Betts, J., Gullone, E., y Allen, J. (2009). An examination of emotion regulation, temperament, and parenting style as potential predictors of adolescent depression risk status: A correlational study. British Journal of Developmental Psychology, 27, 473-485.

Berking, M., Margraf, M., Ebert, D., Wupperman, P., Hofmann, S. G., y Junghanns, K. (2011). Deficits in emotion-regulation skills predict alcohol use during and after cognitive-behavioral therapy for alcohol dependence. Journal of consulting and clinical psychology, 79, 307. doi: $10.1037 / \mathrm{a} 0023421$

Bradley, S. (2000). Affect regulation and the development of psychopathology. New York: Guilford Press.

Buchan, B. D., DeAngelis, D. L., y Levinson, E. M. (2005). A comparison of the web-based and paper-and-pencil versions of the Career Key Interest Inventory with a sample of university women. Journal of Employment Counseling, 42, 39-46. 
Buchanan, T., y Smith, J. L. (1999). Using the Internet for psychological research: Personality testing on the World Wide Web. British Journal of Psychology, 90, 125-144.

Caplan, S. E. (2010). Theory and measurement of generalized problematic Internet use: A two-step approach. Computers In Human Behavior, 26, 1089-1097. doi:10.1016/j. chb.2010.03.012

Carbonell, X. (2014). La adicción a los videojuegos en el DSM-5. Adicciones, 26, 91-95.

Carbonell, X., Fúster, H., Chamarro, A., y Oberst, U. (2012). Adicción a Internet y móvil: una revisión de estudios empíricos españoles. Papeles del Psicólogo, 33, 82-89.

Cooper, M.L., Agocha, V.B., y Sheldon, M.S. (2000). A motivational perspective on risky behaviors: The role of personality and affect regulatory processes. Journal of Personality, 68, 1059-1088.

Cooper, M.L., Wood, P.K., Orcutt, H.K., y Albino, A.W. (2003). Personality and the predisposition to engage in risky or problem behaviors during adolescence. Journal of Personality and Social Psychology, 84, 390-410. doi:10.1037/0022-3514.84.2.390

Curry, L. A., y Youngblade, L. M. (2006). Negative affect, risk perception, and adolescent risk behavior. Journal of applied developmental psychology, 27, 468-485.

d'Acremont, M. U., y Van der Linden, M. (2007). How is impulsivity related to depression in adolescence? Evidence from a French validation of the Cognitive Emotion Regulation Questionnaire. Journal of Adolescence, 30, 271-282.

Derogatis, L. R. (2002). SCL-90-R. Cuestionario de 90 sintomas. Manual. Madrid: Tea Ediciones.

Donati, M., Chiesi, F., y Primi, C. (2013). A model to explain at-risk/problem gambling among male and female adolescents: Gender similarities and differences. Journal of Adolescence, 36, 129-137. doi:10.1016/j.adolescence.2012.10.001

Durkee, T., Kaess, M., Carli, V., Parzer, P., Despalins, R., Floderus, B.,... Wasserman, D. (2012). Prevalence of pathological Internet use among adolescents in Europe: demographic and social factors. Addiction, 107, 2010-2022. doi: 10.1111/j.1360-0443.2012.03946.x.

García del Castillo, J. A., y Días, P. (2007). Análisis relacional entre los factores de protección, resiliencia, autorregulación y consumo de drogas. Salud y drogas, 7, 309-332.

Gibbons, F. X., Kingsbury, J. H., y Gerrard, M. (2012). Social-psychological theories and adolescent health risk behavior. Social and Personality Psychology Compass, 6, 170183. doi:10.1111/j.1751-9004.2011.00412.x

Gratz, K.L., y Roemer, L. (2004). Multidimensional assessment of emotion regulation and dysregulation: Development, factor structure, and initial validation of the difficulties in emotion regulation scale. Journal of Psychopathology and Behavioral Assessment, 26, 41-54.
Griffiths, M. D., y Parke, J. (2010). Adolescent gambling on the Internet: A review. International Journal of Adolescent Medicine and Health, 22, 59-75.

Haagsma, M. C., Caplan, S. E., Peters, O., y Pieterse, M. E. (2013). A cognitive-behavioral model of problematic online gaming in adolescents aged 12-22 years. Computers in Human Behavior, 29, 202-209. doi:10.1016/j. chb.2012.08.006

Hastings, E. C., Karas, T. L., Winsler, A., Way, E., Madigan, A., y Tyler, S. (2009). Young children's video/computer game use: Relations with school performance and behavior. Issues in Mental Health Nursing, 30, 638-649. doi:10.1080/01612840903050414

Hayes, A. F. (2013). Introduction to Mediation, Moderation, and Conditional Process Analysis. New York: Guilford Press.

Hervás, G. y Jódar, R. (2008). Adaptación al castellano de la Escala de Dificultades en la Regulación Emocional. Clinica y Salud, 19, 139-156.

Hessler, D., y Katz, L. F. (2010). Brief report: Associations between emotional competence and adolescent risky behavior. Journal of Adolescence, 33, 241-246.

Hsieh, M., y Stright, A. D. (2012). Adolescents' emotion regulation strategies, self-concept, and internalizing problems. The Journal of Early Adolescence, 32, 876-901.

Kaess, M., Durkee, T., Brunner, R., Carli, V., Parzer, P., Wasserman, C.,... \& Wasserman, D. (2014). Pathological Internet use among European adolescents: psychopathology and self-destructive behaviours. European Child \& Adolescent Psychiatry, 1-10. doi:10.1007/s00787-0140562-7

Karim, R., y Chaudhri, P. (2012). Behavioral addictions: An overview. Journal of Psychoactive Drugs, 44, 5-17. doi:10.10 80/02791072.2012.662859

Keenan, K. (2000). Emotion dysregulation as a risk factor for child psychopathology. Clinical Psychology: Science and Practice, 7, 418-434. doi:10.1093/clipsy/7.4.418

Lougheed, J. P., y Hollenstein, T. (2012). A limited repertoire of emotion regulation strategies is associated with internalizing problems in adolescence. Social Development, 21, 704-721.

Maguire, E. R., Wells, W., y Katz, C. M. (2011). Measuring community risk and protective factors for adolescent problem behaviors evidence from a developing nation. Journal of Research in Crime and Delinquency, 48, 594620.

McLaughlin, K. A., Hatzenbuehler, M. L., Mennin, D. S., y Nolen-Hoeksema, S. (2011). Emotion dysregulation and adolescent psychopathology: A prospective study. Behaviour Research And Therapy, 49, 544-554. doi:10.1016/j. brat.2011.06.003

Miller, D. J., Vachon, D. D., y Aalsma, M. C. (2012). Negative affect and emotion dysregulation conditional relations with violence and risky sexual behavior in a sample 
of justice-involved adolescents. Criminal Justice and Behavior, 39, 1316-1327. doi:10.1177/0093854812448784

Mullin, B. C., y Hinshaw, S. P. (2007). Emotion regulation and externalizing disorders in children and adolescents. In J.J. Gross (Ed.), Handbook of emotion regulation (pp. 523-541). New York: Guilford Press.

Pedrero Pérez, E. J., Rodríguez Monje, M. T., Gallardo Alonso, F., Fernández Girón, M., Pérez López, M., y Chicharro Romero, J. (2007). Validación de un instrumento para la detección de trastornos del control de impulsos y adicciones: el MULTICAGE CAD-4. Trastornos Adictivos, 9, 269-278.

Petry, N. M. (2006). Internet gambling: An emerging concern in family practice medicine? Family Practice, 23, 421-426. doi:10.1093/fampra/cml005

Pisani, A. R., Wyman, P. A., Petrova, M., Schmeelk-Cone, K., Goldston, D. B., Xia, Y., y Gould, M. S. (2013). Emotion regulation difficulties, youth-adult relationships, and suicide attempts among high school students in underserved communities. Journal of Youth And Adolescence, 42, 807-820.

Puerta-Cortés, D. X., y Carbonell, X. (2014). El modelo de los cinco grandes factores de personalidad y el uso problemático de Internet en jóvenes colombianos. Adicciones, 26, 54-61.

Raisamo, S., Halme, J., Murto, A., y Lintonen, T. (2013). Gambling-related harms among adolescents: a population-based study. Journal of Gambling Studies, 29, 151-159. doi:10.1007/s10899-012-9298-9

Ruiz-Olivares, R., Lucena, V., Pino, M., y Herruzo, J. (2010). Análisis de comportamientos relacionados con el uso/ abuso de Internet, teléfono móvil, compras y juego en estudiantes universitarios. Adicciones, 22, 301-310.

Schreiber, L. N., Grant, J. E., y Odlaug, B. L. (2012). Emotion regulation and impulsivity in young adults. Journal of Psychiatric Research, 46, 651-658. doi:10.1016/j.jpsychires.2012.02.005

Shead, N., Callan, M. J., y Hodgins, D. C. (2008). Probability discounting among gamblers: Differences across problem gambling severity and affect-regulation expectancies. Personality and Individual Differences, 45, 536-541. doi:10.1016/j.paid.2008.06.008

Silk, J.S., Steinberg, L., y Morris, A.S. (2003). Adolescents' emotion regulation in life: Links to depressive symptoms and problem behavior. Child Development, 74, 1869 1880. doi:10.1046/j.1467-8624.2003.00643.x

Southam-Gerow, M. A., y Kendall, P. C. (2002). Emotion regulation and understanding: Implications for child psychopathology and therapy. Clinical psychology review, 22, 189-222. doi:10.1016/S0272-7358(01)00087-3

Szwedo, D. (2013). The development of emotion regulation strategies during adolescence and their associations with youths' psychological adjustment in early adulthood. Dissertation Abstracts International, 73, 7-B.
Tice, D. M., Bratslavsky, E., y Baumeister, R. F. (2001). Emotional distress regulation takes precedence over impulse control: If you feel bad, do it!. Journal of Personality and Social Psychology, 80, 53-67. doi:10.1037/0022-3514.80.1.53

Tsitsika, A., Critselis, E., Janikian, M., Kormas, G., y Kafetzis, D. A. (2011). Association between Internet gambling and problematic Internet use among adolescents. Journal of Gambling Studies, 27, 389-400. doi:10.1007/s10899010-9223-z

Van Rooij, A. J., Schoenmakers, T. M., Vermulst, A. A., Van Den Eijnden, R. J. J., y Van De Mheen, D. (2011) . Online video game addiction: identification of addicted adolescent gamers. Addiction, 106, 205-212. doi:10.1111/ j.1360-0443.2010.03104.x

Vasilev, C. A., Crowell, S. E., Beauchaine, T. P., Mead, H. K., y Gatzke-Kopp, L. M. (2009). Correspondence between physiological and self-report measures of emotion dysregulation: A longitudinal investigation of youth with and without psychopathology. Journal of Child Psychology and Psychiatry, 50, 1357-1364. doi:10.1111/j.14697610.2009.02172.x

Walther, B., Morgenstern, M., y Hanewinkel, R. (2012). Co-occurrence of addictive behaviours: Personality factors related to substance use, gambling and computer gaming. European Addiction Research, 18, 167-174. doi:10.1159/000335662

Williams, A. D., Grisham, J. R., Erskine, A., y Cassedy, E. (2012). Deficits in emotion regulation associated with pathological gambling. British Journal Of Clinical Psychology, 51, 223-238. doi:10.1111/j.2044-8260.2011.02022.x 